\title{
Hypertrophic cardiomyopathy without asymmetric hypertrophy
}

\author{
TADASHI KOIDE, TAKEO NARITA, SEIICHI SUMINO \\ From the Second Department of Internal Medicine and the Department of Pathology, Faculty of Medicine, University \\ of Tokyo, Tokyo, fapan
}

SUMMARY A 49-year-old woman with congestive heart failure and heart block died of cerebral embolism. Clinical and echocardiographic findings suggested a diagnosis of atypical dilated cardiomyopathy with predominantly right ventricular involvement. At necropsy, all the cardiac chambers were slightly dilated and the interventricular septum and the left ventricular wall were of normal thickness and symmetry. Histological examination, however, disclosed extensive disarray of abnormal myocardial tissue, especially in the interventricular septum. Her father had similar clinical and echocardiographic findings, while one of her brothers had typical hypertrophic cardiomyopathy at necropsy. It is likely that the patient actually had inherited hypertrophic cardiomyopathy. The case illustrates the difficulty in diagnosing hypertrophic cardiomyopathy when based solely on the left ventricular gross anatomy.

It has been generally believed that the characteristic gross anatomical features of the left ventricle in hypertrophic cardiomyopathy are preserved until death, though clinical manifestations terminally may masquerade as dilated cardiomyopathy. ${ }^{1}$ The case we report had findings suggestive of dilated cardiomyopathy on gross anatomy, while histological findings and the findings in her family members suggested a diagnosis of hypertrophic cardiomyopathy.

\section{Case report}

A 39-year-old housewife was admitted on 9 July 1969, because of exertional dyspnoea. Cardiomegaly had been recognised since the age of 34 , when she had acute renal disease. Otherwise, her past history was non-contributory. Her father and four of the eight sibs were affected by cardiomyopathy, and several family members had raised circulating heart antibody titres and type-specific streptococcal antigens. Details of this family have been published previously ${ }^{2}$ and the present case was then case 2. Subsequent echocardiographic examinations in her father (case 1$)^{2}$ disclosed normal left ventricular dimensions, no left ventricular hypertrophy, posterior wall hypokinesis, and paradoxical septal motion (Fig. 1b). Necropsy findings in her brother (case 7) ${ }^{2}$ have been reported as those of symmetric left ventricular hypertrophy and

This work was supported by a grant from the Japanese Ministry of Health and Welfare for the study of cardiomyopathy. myocardial fibrosis. ${ }^{1}$ Haematoxylin-eosin stained sections of the interventricular septum and the left ventricular free wall of this case were reviewed by us. We found myocardial disarray occupying extensive areas of the interventricular septum (70\%) and left ventricular free wall (50\%) (Fig. 2c). A few foci of small round cell infiltration were seen in the endocardium and the myocardium (Fig. 2d).

The present case was a small woman $(148 \mathrm{~cm}$ tall, body weight $53 \mathrm{~kg}$ ). Her heart rate was 68 beats $/ \mathrm{min}$, and her blood pressure $130 / 80 \mathrm{mmHg}$. There was evidence of cardiomegaly on physical examination. Loud third and fourth heart sounds and a grade $2 / 6$ systolic murmur were heard at the apex. The response of the murmur to amyl nitrite inhalation did not suggest the presence of left ventricular outflow tract obstruction. Routine laboratory examinations disclosed a moderate iron deficiency anaemia, but were otherwise normal. There was a global cardiomegaly on the chest $x$-ray film. Electrocardiographic findings were sinus rhythm, left atrial enlargement, left ventricular hypertrophy (SV1: $0.9 \mathrm{mV}, \mathrm{RV} 5: 2.8 \mathrm{mV}$ ), and slight ST depression in leads I, II, III, aVF, and V3-V6. There were no abnormal $Q$ waves. Circulating antiheart antibody was positive at a titre of 1 in 160 and type 18-specific anti-beta-haemolytic streptococcal antibody was positive at 1 in 3072. Dyspnoea diminished with correction of anaemia, but the $x$-ray film and electrocardiogram were unchanged. She was discharged on 9 August with the diagnosis of non- 
obstructive cardiomyopathy and iron deficiency anaemia.

On 17 January 1972, atrial fibrillation developed and persisted thereafter. Her subsequent course was that of progressive congestive heart failure with cardiomegaly, despite digitalis and diuretics. An echocardiogram was taken in June 1976. The left ventricular dimensions were $54 \mathrm{~mm}$ at end-diastole and $48 \mathrm{~mm}$ at end-systole. Both the interventricular septum and left ventricular free wall were $12 \mathrm{~mm}$ thick. All the other cardiac chambers were moderately dilated and there was a moderate pericardial effusion. A pansystolic murmur with Rivero-Carvallo's sign was heard along the left sternal border. The patient was considered to have a dilated cardiomyopathy with predominant right ventricular involvement and functional tricuspid regurgitation. Circulating antiheart antibody was present at a titre of 1 in 40 which was the last positive value obtained.

A permanent pacemaker was implanted on 25 January 1978, because of complete heart block, which subsequently altered to persistent atrial flutter with variable atrioventricular block. She was now confined to bed because of her low output state.

Bilateral leg thrombophlebitis developed in August 1978. A lung scan disclosed a few small perfusion defects, but there was no infarction shadow on the chest $x$-ray film. Follow-up echocardiograms showed a decrease in left ventricular dimensions to normal values, but otherwise there was no change compared with the 1976 findings (Fig. 1a). She died of cerebral embolism on 6 October 1979.

At necropsy, heart weight was $330 \mathrm{~g}$. The left ventricle was slightly dilated, the major and the minor axes of the cavity being 7 and $4.5 \mathrm{~cm}$, respectively, and the thickness of the wall being 10 to $12 \mathrm{~mm}$. There was no asymmetry of the left ventricular wall, as shown in Fig. 2a. The other cardiac chambers were dilated, and there were jet lesions in the atria suggesting atrioventricular valve regurgitation. The valves were normal. A screw electrode was fixed on the right ventricular wall, around which there was fibrotic adhesion of the pericardium. Otherwise, there was no evidence of pericarditis. The aorta and the coronary arteries were normal. The lungs were also normal. In the brain, a large infarct in and around the internal capsule and a few haemorrhagic foci in the pons were seen. Old and fresh infarcts were seen in the right kidney and the spleen. The source of the systemic emboli was obscure, since there was no thrombus in the cardiac chambers or the pulmonary veins. Systemic veins were examined to the level of the femoral and subclavian, and no thrombus found. The liver showed evidence of cardiac cirrhosis.

On histology, myocardial disarray was seen throughout the left ventricular wall, and was exten-
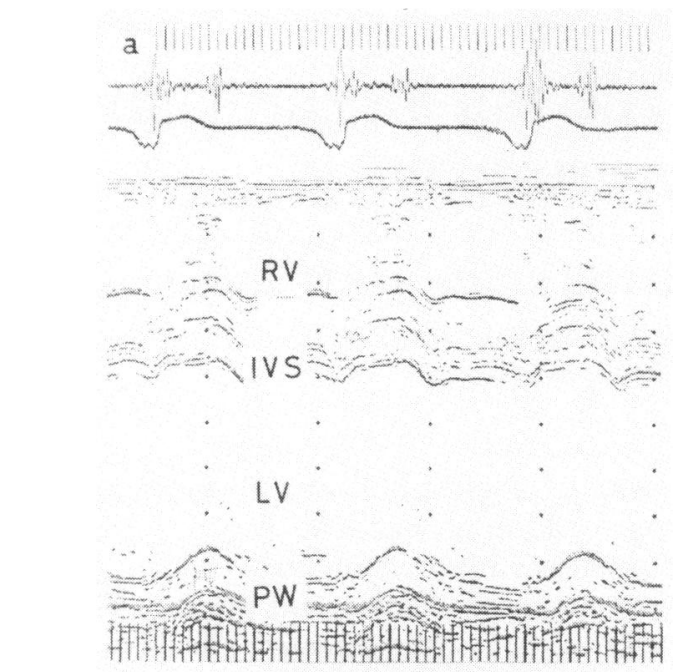

b

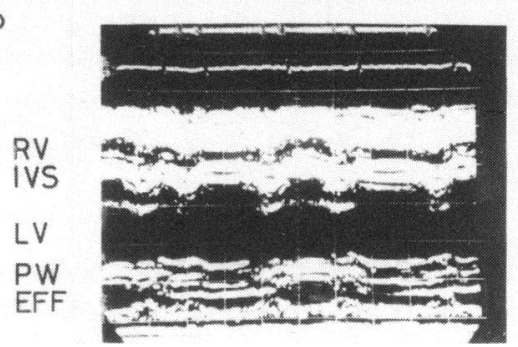

Fig. 1 Echocardiograms of the present case (a) and her father (b). In (a), which was taken in March 1978, both the interventricular septum (IVS) and the left ventricular posterior wall (PW) measured 6 to $7 \mathrm{~mm}$. End-diastolic dimension of the right ventricle $(R V)$ was $30 \mathrm{~mm}$, while that of the left ventricle (LV) was $45 \mathrm{~mm}$. Motion of the interventricular septum was paradoxical. An echocardiogram of her father $(b)$ was essentially similar to (a), showing normal thickness of the interventricular septum $(10 \mathrm{~mm})$ and posterior wall $(10 \mathrm{~mm})$, normal left ventricular dimension (45 mm), and paradoxical interventricular septal motion. Posterior wall motion was hypokinetic and there was pericardical effusion (EFF).

sive particularly in the interventricular septum, occupying about $70 \%$ of the equatorial transsectional area (Fig. 2b). Disarray was prominent also in the anterolateral left ventricular wall and the anterior right ventricular wall. Many whorls were seen. Interstitial fibrosis was also prominent. Myocardial fibres in the disorganised tissue were branched in peculiar forms and were variable in size. Many fibres were thick, some being $90 \mu \mathrm{m}$, but were short and interrupted by fibrous tissue. There were areas of myocytolysis, leaving only the sarcolemmal sheath. Nuclei were frequently hypertrophied and of bizarre shape. Nuclear halo was present, but was not extensive. The intramyocardial small coronary arteries 


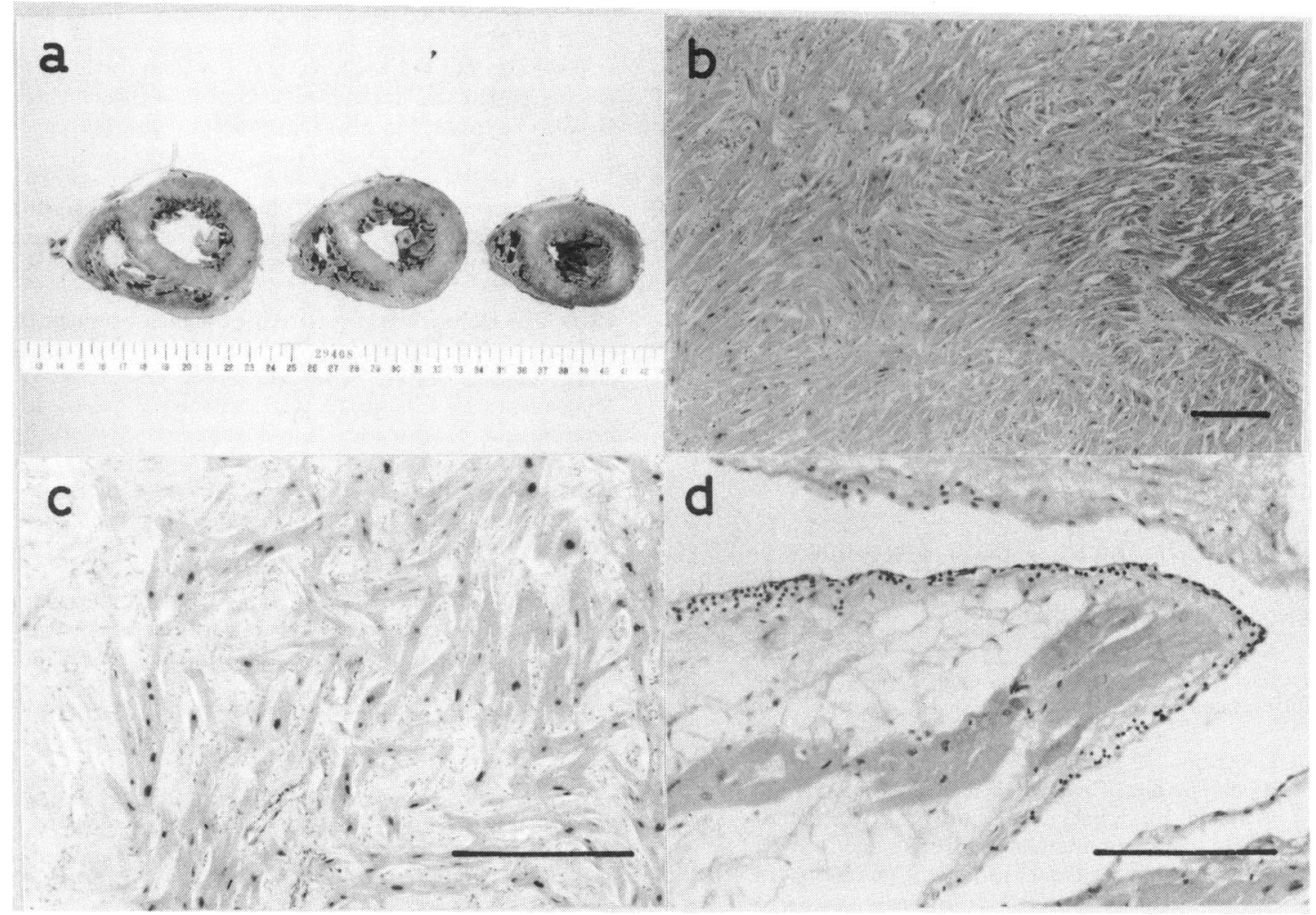

Fig. 2 Necropsyfindings in the present case $(a, b)$ and in her brother $(c, d)$. In $(a)$, sequential cross-sections of the heart after fixation are shown. There is no asymmetric septal hypertrophy. The interventricular septum, however, contains large amounts of disarrayed myocardial fibres as in (b) (haematoxylin-eosin stain). Many myocardial fibres are hypertrophied and nuclei are bizarre in shape. Prominent myocardial disarray is also seen in her brother's interventricular septuon (c) (haematoxylin-eosin stain). A few foci of small round cell infiltration are seen in the endocardium (d) (haematoxylin-eosin stain) and the myocardium in the latter, but not in the present case. The bars in the figure show $500 \mu \mathrm{m}$ in (b) and (c), and $200 \mu \mathrm{m}$ in (d).

were occasionally narrowed with medial hypertrophy. There was no inflammatory cell infiltration.

\section{Discussion}

Myocardial disarray in the interventricular septum, when extensive as in our patient, has been considered a specific finding of hypertrophic cardiomyopathy. ${ }^{34}$ Extreme thickness of myocardial fibres, extensive fibrosis, bizarre muscle nuclei, disappearing myocardial fibres with nuclear halos, whorls, and short runs of fibres, in combination, are highly suggestive of the diagnosis of hypertrophic cardiomyopathy. ${ }^{56}$ The findings of hypertrophic cardiomyopathy in her brother at necropsy, and the overall family history suggesting autosomal dominant inheritance of the cardiomyopathy were also consistent with this diagnosis. ${ }^{78}$

On the other hand, asymmetric hypertrophy was not seen on echocardiograms, and the necropsy findings were those (macroscopically) of dilated cardiomyopathy. The clinical diagnosis of hypertrophic cardiomyopathy is based on the recognition of undue left ventricular hypertrophy, particularly in the form of asymmetric septal hypertrophy. ${ }^{910}$ Asymmetric septal hypertrophy has been seen even terminally in hypertrophic cardiomyopathy with clinical manifestations masquerading as dilated cardiomyopathy, ${ }^{1}$ and even after the development of left ventricular dilatation. ${ }^{11}$ Asymmetric hypertrophy, however, is not strictly a sine qua non for the diagnosis of hypertrophic cardiomyopathy, since there are occasional cases with left ventricular hypertrophy which are symmetrical. ${ }^{12}$ If terminal ventricular dilatation occurs in such hearts, the gross anatomical findings may mimic those of dilated cardiomyopathy. Olsen ${ }^{5}$ has described five cases resembling dilated cardiomyopathy, but indistinguishable from hypertrophic cardiomyopathy histologically. None of the five had a definite family history of hypertrophic cardiomyopathy. In the present 
case, the existence of necropsy-proven hypertrophic cardiomyopathy in the family members suggests that the patient's cardiomyopathy is within the spectrum of this disease.

The relation between the immunological and cardiological abnormalities is obscure. According to Das et al., ${ }^{13}$ antiheart antibody is frequently positive in hypertrophic cardiomyopathy, and the titre is high especially in the stage of progression. In the present case, however, antiheart antibody was positive only in the early stages and did not correlate with the clinical course. Immunological studies were not carried out in her brother, though the focal cell infiltration in his heart may suggest a possible link between the immunological abnormalities and the cardiomyopathy in the family.

We thank Dr Akihiro Takatsu, Department of Forensic Medicine, University of Tokyo, for his cooperation on the family study.

\section{References}

1 Goodwin JF, Oakley CM. Editorial: The cardiomyopathies. Br Heart $\mathcal{F}$ 1972; 34: 545-52.

2 Machida K, Iguchi K, Saito Y, et al. Familial cardiomyopathy: immunological studies and review of literatures on autopsied cases in Japan. Fpn Heart $\mathcal{F} 1971 ; 12$ : 40-9.

3 Maron BJ, Reberts WC. Quantitative analysis of cardiac muscle cell disorganisation in the ventricular septum of patients with hypertrophic cardiomyopathy. Circulation 1979; 59: 689-706.

4 Maron BJ, Sato N, Roberts WC, Edwards JE, Chandra RS. Quantitative analysis of cardiac muscle cell disorganisation in the ventricular septum: comparison of fetuses and infants with and without congenital heart disease and patients with hypertrophic cardiomyopathy. Circulation 1979; 60: 685-96.

5 Olsen EGJ. Morbid anatomy and histology in hypertrophic obstructive cardiomyopathy. In: Wolstenholme GEW, O'Connor M, eds. Hypertrophic obstructive cardiomyopathy. London: Churchill, 1971: 183-91.

6 Ferrans VJ, Morrow AG, Roberts WC. Myocardial ultrastructure in idiopathic hypertrophic subaortic stenosis: a study of operatively excised left ventricular outflow tract muscle in 14 patients. Circulation 1972; 45: 769-92.

7 Clark CE, Henry WL, Epstein SE. Familial prevalence and genetic transmission of idiopathic hypertrophic subaortic stenosis. $N$ Engl f Med 1973; 289: 709-14.

8 Yamaguchi M, Toshima H, Yanase T. Genetic heterogeneity of idiopathic cardiomyopathies. Fpn Circ $\mathcal{Y}$ 1978; 42: 1131-2.

9 Henry WL, Clark CE, Epstein SE. Asymmetric septal hypertrophy: echocardiographic identification of the pathognomonic anatomic abnormality of IHSS. Circulation 1973; 47: 225-33.

10 Redwood DR, Scherer JL, Epstein SE. Biventricular cineangiography in the evaluation of patients with asymmetric septal hypertrophy. Circulation 1974; 49: 1116-21.

11 ten Cate FJ, Roelandt J. Progression to left ventricular dilatation in patients with hypertrophic obstructive cardiomyopathy. Am Heart $\mathcal{f}$ 1979; 97: 762-5.

12 Davies MJ. The cardiomyopathies. In: Pomerance A, Davies MJ, eds. The pathology of the heart. Oxford: Blackwell, 1975: 211-50.

13 Das SK, Cassidy JT, Dodson VN, Willis PW. Antiheart antibody in idiopathic hypertrophic subaortic stenosis. Br Heart f 1973; 35: 965-9.

Requests for reprints to Dr Tadashi Koide, The Second Department of Internal Medicine, University of Tokyo Hospitals, 7-3-1 Hongo, Bunkyo-ku, Tokyo 113, Japan. 